\title{
Pattern of Electrolytes Including Calcium and Magnesium Imbalance with Etiology Assessment in Patients Admitted in Endocrine Department of a Tertiary Care Hospital
}

\author{
Shahin Ibn Rahman ${ }^{1}$, Milton Barua ${ }^{2,}$, , Nausher Azimul Huq ${ }^{3}$, Bimol Kumar Agarwala ${ }^{3}$, \\ Muhbubunnesa Sadia ${ }^{4}$ \\ ${ }^{1}$ Department of Endocrinology, Dhaka National Medical Institute Hospital, Dhaka \\ ${ }^{2}$ Department of Medicine, Sadar Hospital, Khagrachari \\ ${ }^{3}$ Department of Endocrinology, Dhaka National Medical College, Dhaka \\ ${ }^{4}$ Department of Endocrinology, Dhaka National Medical Institute Hospital, Dhaka
}

Email address:

dr.miltonbarua@gamil.com (M. Barua)

${ }^{*}$ Corresponding author

To cite this article:

Shahin Ibn Rahman, Milton Barua, Nausher Azimul Huq, Bimol Kumar Agarwala, Muhbubunnesa Sadia. Pattern of Electrolytes Including Calcium and Magnesium Imbalance with Etiology Assessment in Patients Admitted in Endocrine Department of a Tertiary Care Hospital. International Journal of Diabetes and Endocrinology. Vol. 5, No. 4, 2020, pp. 71-76. doi: 10.11648/j.ijde.20200504.14

Received: November 10, 2020; Accepted: November 25, 2020; Published: December 22, 2020

\begin{abstract}
Objectives: Several endocrine disorders have marked effects on fluid, electrolyte, and acid-base homeostasis including diabetic ketoacidosis, hyperglycemic hyperosmolar state, and acute adrenal crisis etc. An understanding of the etiology behind the development of these electrolytes along with calcium and magnesium imbalance helps to guide therapy and improves the clinical outcome. The aim of this study to see the pattern of electrolytes, calcium and magnesium imbalance with etiology assessment in patients admitted in endocrine department of a tertiary care hospital. Study design and methods: This cross sectional observational study was carried out from January 2018 to December 2018, at in-patient department of Endocrinology, BIRDEM General Hospital, Dhaka. Diagnosed (old and new) cases of diabetic and other endocrine disorders having electrolytes, calcium and magnesium imbalance were approached for sinclusion of the study. Sampling technique was purposively selected focusing on demographic profile and diagnosis of the disease. Results: Among the 100 participants, mean age of the study population was $46.26 \pm 16.97$ years, ranging from 14 to 75 years. There were $50 \%$-male and $50 \%$-female. In result the most common electrolyte imbalance was hyponatremia $(36 \%)$ that was more in type-2 DM patients $(\mathrm{n}=25)$ then in Addison's disease $(\mathrm{n}=4)$ and rest are in other specific form of DM $(\mathrm{n}=3)$, type-1 DM ( $\mathrm{n}=2)$, DM with hypoparathyroidism $(\mathrm{n}=1)$ and hypoparathyroidism $(\mathrm{n}=1)$ which may be due to SIADH. Followed by hypokalemia $(14 \%)$ which mostly encountered in type-2 DM patient $(n=6)$ due to diuretics and in Conn's syndrome $(n=4)$, hyperkalemia $(10 \%)$ which mostly observed in type-1 DM patient $(\mathrm{n}=4)$ due to DKA, and hypercalcemia $(8 \%)$ was found in non-diabetic endocrine disorders. Hypernatremia, hypocalcemia and hypomagnesemia were present in $7 \%$, in $6 \%$, and $6 \%$ cases, respectively. The most common precipitating cause of these electrolyte imbalances was vomiting (30\%) due to different causes like urinary tract infection; acute gastritis and pancreatitis. Diuretic (Loop \& Thiazide) therapy $(10 \%)$ and HHS (8\%) were the second and third most common cause behind these electrolytes imbalance Conclusion: In conclusion data obtained in this study showed hyponatremia was the most common findings which more observed in diabetic patients. As vomiting is the most common cause behind this so any diabetic patients either present with vomiting or any other illness should routinely advice electrolytes along with magnesium as hypomagnesemia also more observed in this group. To find out the cause of hypokalemia in non-diabetic patients should evaluate the Conn's syndrome and don't forget to measure the parathyroid hormone in hypercalcemic patient. Further study is needed to find out the causes of vomiting.
\end{abstract}

Keywords: Electrolytes, Diabetes Mellitus, Addison's Disease, Hyperparathyroidism 


\section{Introduction}

Molecules that dissociate in water form cations (positively charged ions) and anions (negatively charged ions) are known as electrolytes which facilitate conduction of electrical current. $\mathrm{Na}+, \mathrm{K}+, \mathrm{Cl}-$ and bicarbonate are examples of electrolytes [1].

Electrolyte balance is regulated by hormones (such as $\mathrm{ADH}$, aldosterone and $\mathrm{PTH}$ ) and in general by the kidneys. The most common electrolyte imbalances are hypo- and hyper-states of sodium, potassium, chloride, calcium, and magnesium. The kidneys are the main organ which is responsible for maintaining the electrolyte balance in the healthy individuals [2]. Besides the kidneys, the other mechanisms which are involved in the regulation of fluid and electrolyte balance, such as hormones; like antidiuretic hormone, aldosterone, cortisol, thyroid and parathyroid hormone, and various other factors such as physiological stress and age also play important roles in the regulation of electrolyte balance [3, 4]. Among the diabetes and endocrine disorders, uncontrolled diabetes mellitus (DM) is most common causes of admission in our endocrinology department of BIRDEM General Hospital. Diabetes mellitus (DM) is rapidly emerging as an important cause of mortality and morbidity in developing countries [5].

\section{Methods and Materials}

This cross sectional observational study was carried out from January 2018 to December 2018, at in-patient department of Endocrinology, BIRDEM General Hospital, Dhaka. Diagnosed (old and new) cases of diabetic and other endocrine disorders having electrolytes, calcium and magnesium imbalance were approached for inclusion of the study. Sampling technique was purposively selected focusing on demographic profile and diagnosis of the disease.

\section{Results}

Table 1. Demographic profile of study participants $(n=100)$.

\begin{tabular}{|c|c|}
\hline Demographic variable & Percent (\%) \\
\hline \multicolumn{2}{|l|}{ Age group } \\
\hline $14-25$ & 16 \\
\hline $26-35$ & 10 \\
\hline $36-45$ & 26 \\
\hline $46-55$ & 12 \\
\hline $56-65$ & 16 \\
\hline $66-75$ & 20 \\
\hline Male & 50 \\
\hline Female & 50 \\
\hline \multicolumn{2}{|l|}{ Occupation } \\
\hline Student & 8 \\
\hline Businessman & 18 \\
\hline Day labour & 2 \\
\hline Housewife & 42 \\
\hline
\end{tabular}

Table 2. Past history of endocrine disease of study participants $(n=100)$.

\begin{tabular}{ll}
\hline Risk factors & Percent \\
\hline Addison's disease & 4 \\
Conn's syndrome & 2 \\
Diabetes Insipidus & 2 \\
Diabetes Mellitus & 6 \\
FCPD & 1 \\
Hypothyroidism & 8 \\
PCOS & 2 \\
Panhypopituitarism & 2 \\
\hline
\end{tabular}

Table 3. Diseases of study participants $(n=100)$.

\begin{tabular}{ll}
\hline Diagnosis & Percent (\%) \\
\hline Diabetic diseases & 58 \\
Type 2 DM & 43 \\
Type 1 DM & 10 \\
FCPD & 1 \\
Other forms of diabetes & 4 \\
Non-diabetic diseases & 42 \\
\hline
\end{tabular}




\begin{tabular}{ll}
\hline Diagnosis & Percent (\%) \\
\hline Hypoparathyroidism & 8 \\
Hyperparathyroidism & 6 \\
Diabetes Insipidus & 5 \\
Addison's disease & 5 \\
Conn's syndrome & 4 \\
Adrenal crisis & 2 \\
Cushing's syndrome & 2 \\
Hypercalcemia & 2 \\
Panhypopituitarism & 2 \\
PCOS & 2 \\
Vitamin D deficiency & 2 \\
Adrenal insufficiency & 2 \\
\hline
\end{tabular}

\section{ELECTROLYTES,CALCIUM \& MAGNESIUM IMBALANCE}

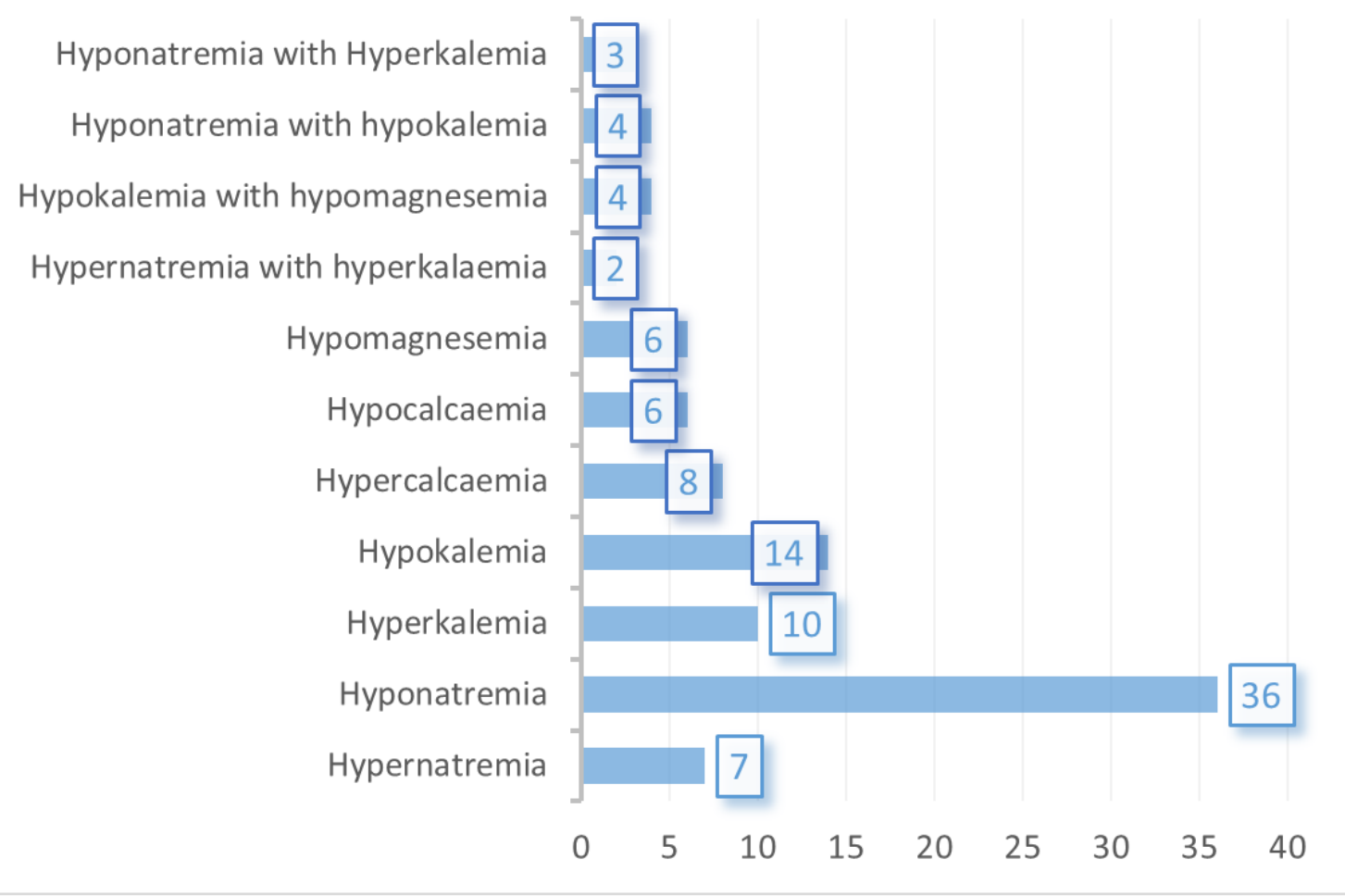

Figure 1. Pattern of electrolytes, calcium and magnesium imbalance in endocrine department $(n=100)$.

Table 4. Pattern of electrolytes, calcium and magnesium imbalance in diabetic and non-diabetic disorders $(n=100)$.

\begin{tabular}{llll}
\hline \multirow{2}{*}{ Electrolyte/micronutrient imbalance } & \multicolumn{2}{l}{ Disorders } & \multirow{2}{*}{ P value } \\
\cline { 2 - 3 } & Diabetic (n=58) & Non-diabetic (n=42) & $\mathbf{N}$ (\%) \\
\cline { 2 - 3 } & $\mathbf{N ~ ( \% )}$ & $5(11.9)$ & 0.102 \\
Hypernatremia & $2(3.4)$ & $6(14.3)$ & $<0.001$ \\
Hyponatremia & $30(51.7)$ & $4(9.5)$ & 0.893 \\
Hyperkalemia & $6(10.3)$ & $8(19.0)$ & 0.216 \\
Hypokalemia & $6(10.3)$ & $8(19)$ & 0.001 \\
Hypercalcaemia & 0 & $6(14.3)$ & 0.003 \\
Hypocalcaemia & 0 & 0 & 0.032 \\
Hypomagnesemia & $6(10.3)$ & 0 & 0.224 \\
Hyponatremia with hyperkalemia & $2(3.4)$ & $2(4.8)$ & 0.741 \\
Hyponatremia with hypokalemia & $2(3.4)$ & 0 & 0.082 \\
Hypokalemia with hypomagnesemia & $4(6.9)$ & $3(7.1)$ & 0.039 \\
Hypernatremia with hyperkalemia & 0 & & \\
\hline
\end{tabular}

P value determined by Chi-squared test. 
Table 5. Pattern of electrolytes, calcium and magnesium imbalance in diabetic patients $(n=58)$

\begin{tabular}{|c|c|c|c|c|c|}
\hline \multirow{4}{*}{$\begin{array}{l}\text { Electrolytes, calcium \& magnesium } \\
\text { imbalance }\end{array}$} & \multicolumn{4}{|c|}{ Diabetic disorder } & \multirow{4}{*}{ P value } \\
\hline & Type 1 DM & \multirow{2}{*}{$\begin{array}{l}\text { Type } 2 \text { DM } \\
(n=43)\end{array}$} & \multirow{2}{*}{$\begin{array}{l}\text { FCPD } \\
(n=1) \\
\end{array}$} & \multirow{3}{*}{$\begin{array}{l}\text { Other forms } \\
\begin{array}{l}(\mathrm{n}=4) \\
\mathrm{N}(\%)\end{array}\end{array}$} & \\
\hline & \multirow{2}{*}{$\begin{array}{l}(\mathrm{n}=10) \\
\mathrm{N}(\%)\end{array}$} & & & & \\
\hline & & N (\%) & $\mathbf{N}(\%)$ & & \\
\hline Hypernatremia & 0 & $2(4.7)$ & 0 & 0 & 0.868 \\
\hline Hyponatremia & $2(20)$ & $25(58.1)$ & $1(100)$ & $2(50)$ & 0.128 \\
\hline Hyperkalemia & $4(40)$ & $2(4.7)$ & 0 & 0 & 0.009 \\
\hline Hypokalemia & 0 & $6(14)$ & 0 & 0 & 0.506 \\
\hline Hypomagnesemia & $2(20)$ & $4(9.3)$ & 0 & 0 & 0.652 \\
\hline Hypernatremia with hyperkalemia & $2(20)$ & 0 & 0 & 0 & 0.019 \\
\hline Hyponatremia with hypokalemia & 0 & $2(4.7)$ & 0 & 0 & 0.868 \\
\hline Hypokalemia with hypomagnesemia & 0 & $2(4.7)$ & 0 & $2(50)$ & 0.005 \\
\hline
\end{tabular}

DM: Diabetes mellitus; DI: Diabetes insipidus; FCPD: Fibrocalcific pancreatic disease, P value determined by Chi-squared test.

Table 6. Pattern of electrolytes, $\mathrm{Ca}^{2+}$ and $\mathrm{Mg}^{2+}$ imbalance in non-diabetic patients and others $(n=42)$.

\begin{tabular}{|c|c|c|c|c|c|c|}
\hline \multirow{2}{*}{ Non-diabetic disorders } & \multirow{2}{*}{$\mathbf{N}$} & \multicolumn{5}{|c|}{ Electrolytes, $\mathrm{Ca}^{2+} \& \mathrm{Mg}^{2+}$ imbalance } \\
\hline & & Hypernatremia & Hyponatremia & Hyperkalemia & Hypokalemia & Hypercalcemia \\
\hline Addison's disease & 5 & 0 & 0 & $2(40)$ & 0 & 0 \\
\hline Adrenal crisis & 2 & 0 & $2(100)$ & 0 & 0 & 0 \\
\hline Adrenal insufficiency & 2 & 0 & $2(100)$ & 0 & 0 & 0 \\
\hline Conn's syndrome & 4 & 0 & 0 & 0 & $4(100)$ & 0 \\
\hline Cushing's syndrome & 2 & 0 & 0 & 0 & 0 & 0 \\
\hline Diabetes Insipidus & 5 & $5(100)$ & 0 & 0 & 0 & 0 \\
\hline Hyperparathyroidism & 6 & 0 & 0 & 0 & 0 & $6(100)$ \\
\hline Hypoparathyroidism & 8 & 0 & $2(25)$ & 0 & $2(25)$ & 0 \\
\hline Vitamin D deficiency & 2 & 0 & 0 & 0 & 0 & 0 \\
\hline Iatrogenic hypercalcemia & 2 & 0 & 0 & 0 & 0 & $2(100)$ \\
\hline Polycystic ovary syndrome & 2 & 0 & 0 & $2(100)$ & 0 & 0 \\
\hline Panhypopituitarism & 2 & 0 & 0 & 0 & $2(100)$ & 0 \\
\hline $\mathrm{P}$ & & $<0.001$ & 0.002 & 0.003 & 0.001 & $<0.001$ \\
\hline
\end{tabular}

Table 6. Continued.

\begin{tabular}{lllll}
\hline \multirow{2}{*}{ Non-diabetic disorders } & Electrolytes, $\mathbf{C a}^{2+} \boldsymbol{\&} \mathbf{M g}^{2+}$ imbalance & & Hypokalemia with \\
\cline { 2 - 5 } & Hypocalcemia & $\begin{array}{l}\text { Hyponatremia with } \\
\text { hypokalemia }\end{array}$ & $\begin{array}{l}\text { Hypernatremia } \\
\text { with hyperkalemia }\end{array}$ & $\begin{array}{l}\text { Hypomagnesemia } \\
\text { hypomany }\end{array}$ \\
\hline Addison's disease & 0 & $3(60)$ & 0 & 0 \\
Adrenal crisis & 0 & 0 & 0 & 0 \\
Adrenal insufficiency & 0 & 0 & 0 & 0 \\
Conn's syndrome & 0 & 0 & 0 & 0 \\
Cushing's syndrome & 0 & 0 & 0 & $2(100)$ \\
Diabetes Insipidus & 0 & 0 & 0 & 0 \\
Hyperparathyroidism & 0 & 0 & 0 & 0 \\
Hypoparathyroidism & $4(50)$ & 0 & 0 & 0 \\
Vitamin D deficiency & $2(100)$ & 0 & 0 & 0 \\
Iatrogenic hypercalcemia & 0 & 0 & 0 & 0 \\
Polycystic ovary syndrome & 0 & 0 & 0 & 0 \\
Panhypopituitarism & 0 & 0 & 0 & 0.013 \\
P & 0.007 & $<0.001$ & & 0 \\
\hline
\end{tabular}

Table 7. Causes of electrolytes, $\mathrm{Ca}^{2+} \& \mathrm{Mg}^{2+}$ imbalance in study participants $(n=100)$.

\begin{tabular}{ll}
\hline Diagnosis & Percent (\%) \\
\hline Vomiting & 30 \\
Diuretics & 10 \\
Hyperglycemic hyperosmolar state & 8 \\
Diabetic ketoacidosis & 6 \\
Hyperparathyroidism & 6 \\
Hypoparathyroidism & 6 \\
ADH deficiency & 5 \\
Adrenal crisis & 4 \\
Hyperaldosteronism & 4 \\
Excess water intake & 4 \\
Cortisol deficiency & 3 \\
Acute gastroenteritis & 2 \\
\hline
\end{tabular}




\begin{tabular}{ll}
\hline Diagnosis & Percent (\%) \\
\hline Cushing's syndrome & 2 \\
Increased oral intake & 2 \\
In vitro hemolysis & 2 \\
Laxative abuse & 2 \\
SIADH & 2 \\
Vitamin D deficiency & 2 \\
\hline
\end{tabular}

\section{Discussion}

Total 100 patients of electrolyte and $\mathrm{Ca}^{2+}, \mathrm{Mg}^{2+}$ imbalance was included with an average age of $46.26 \pm 16.97$ years. Majority patients were middle aged with an age between 41 60 years $(40 \%)$. Twenty six percent patients were aged between $20-40$ years and $>60$ years. Ahmed et al in their study involving diabetic patients noted that hyponatremia was more common among elderly ( $>60$ years) patients [6]. Haque and her colleagues also noted a prevalence of elderly people among diabetic patients admitted with electrolyte imbalance [7]. On the other hand, middle age bulk in this study was constituted by patients having adrenal disease and parathyroid disease alongside type 1 and $2 \mathrm{DM}$.

The present study found $58 \%$ diabetic, $30 \%$ non-diabetic cases and $12 \%$ cases of diabetes with other endocrine disorders. Among diabetic patients $43 \%$ had type 2 diabetes mellitus. Among non-diabetic patients, parathyroid disorders were relatively common. The most common electrolyte imbalance was hyponatremia (36\%) and this was found in significantly higher proportion among diabetic patients. This is concordant with the findings of Ahmed and colleagues who found $63.3 \%$ cases of hyponatremia among diabetic patients [6]. It was found to be $80 \%$ in a study conducted in one of the largest hospital of Bangladesh mainly for diabetes care named Bangladesh Institute of Research \& Rehabilitation in Diabetes, Endocrine and Metabolic Disorders (BIRDEM) [7].

The most common precipitating cause for electrolyte imbalance was vomiting (30\%). This is also consistent with findings of Ahmed and Haque who reported respectively 54.7\% and $51 \%$ cases of electrolyte imbalance due to vomiting in their study $[6,7]$. In this study most of the diabetic patient suffered from vomiting due to different cause like UTI, acute gastritis \& pancreatitis. In some patients the actual cause of vomiting could not find out.

An important cause of dilutional hyponatremia is SIADH and was found in 2 cases of type 2 diabetes in the present study [8]. Other causes of hyponatremia may be diuretic use which was found in $10 \%$ cases of the present study. In general practice, diuretic use (specially loop diuretic and thiazide diuretic) is the most common cause of hyponatremia [9]. Present study also noted 7\% patients had hypernatremia and $3.4 \%$ of diabetic patients had hypernatremia. This is because usually DKA and HHS is associated with hyponatremia at presentation but may also present with hypernatremia [10].

Hypokalemia was the second most common electrolyte imbalance constituting $14 \%$ of the total cases. Boysen noted that total body potassium and phosphorus depletion often is profound in patients that have DKA and HHS ${ }^{10}$. This may explain the $10.3 \%$ cases of hypokalemia among diabetic patients in this study. Hypokalemia was also found in 4 cases of Conn's syndrome, $2 \%$ cases of panhypopituitarism (one case with DM and one isolated case) due to vomiting and interestingly in two cases of hypoparathyroidism due to laxative abuse. Adrenal steroid excess in Cushing's syndrome and primary hyperaldosteronism (Conn's syndrome) are important renal causes of potassium loss and therefore explains the presence of these cases in the present study [11].

Hyperkalemia alone was found in $10 \%$ cases and was the third most common cause of electrolyte imbalance in this study. Hyperkalemia was found in Addison's disease among non-diabetic cases, in PCOS with DM (due to spironolactone) and in type $2 \mathrm{DM}$ patients due in vitro hemolysis. The second most common cause of electrolyte imbalance was diuretic use $(10 \%)$, including thiazide diuretics and spironolactone. Hyperkalemia was associated with hyponatremia in 3\% cases found in Addison's disease with DM and with hypernatremia in $2 \%$ cases found in HHS (type $2 \mathrm{DM}$ ). Aldosterone deficiency causes loss of sodium and increased potassium in the blood and hyperkalemia in HHS may be due to hyperosmolar state $[12,13]$. Two cases of hyperkalemia associated with PCOS were due to use of spironolactone.

Imbalance in calcium metabolism was exclusively found in non-diabetic patients and in patients with combined diabetes and other endocrine disorders. There were 8 cases of hypercalcemia all of which was associated with hyperparathyroidism. Among 6 cases of hypocalcemia 4 were due to hypoparathyroidism (two isolated case and two cases with DM) and 2 were due vitamin D deficiency alongside diabetes.

In this study total $8 \%$ cases of isolated hypomagnesemia were found. Among them 6\% were isolated cases and 2\% was associated with hypokalemia. Six cases of isolated hypomagnesemia were diabetic which may due to HHS and GI loss. Two cases hypomagnesemia with hypokalemia were non-diabetic (due to Cushing's syndrome). Dasgupta et al studied 150 cases of non-critical type $2 \mathrm{DM}$ and found hypomagnesemia in $11.33 \%$ patients [14].

The reason is among the endocrine and metabolic disorders associated with magnesium deficiency, diabetes mellitus is the most common [15]. Cushing's syndrome cases presented with hypomagnesemia and hypokalaemia simultaneously in the present study. This type of cases were reported in Cushing's syndrome and Pseudo-Cushing's syndrome in previous studies validating the finding of this study [16]. 


\section{Limitations}

1. Data collection primarily confined with the BIRDEM general Hospital

2. Sample size was not representative

3. No follow up was done and it was beyond the scope of the study.

4. Immunological confirmation of type-1 DM cannot be done.

5. Regarding etiology assessment of all vomiting case patients were not evaluated properly.

\section{Conclusion}

Data obtained in this study showed hyponatremia was the most common findings which more observed in diabetic patients. As vomiting is the most common cause behind this so any diabetic patients either present with vomiting or any other illness should routinely advice electrolytes along with magnesium as hypomagnesemia also more observed in this group. To find out the cause of hypokalemia in non-diabetic patients should evaluate the Conn's syndrome and don't forget to measure the parathyroid hormone in hypercalcemic patient. Further study is needed to find out the causes of vomiting.

\section{References}

[1] Devlin, T. M. (2010) Textbook of Biochemistry with Clinical Correlations. 7 th edi. p. 5.

[2] Bockenkamp, B. and Vyas, H. (2003) 'Understanding and managing acute fluid and electrolyte disturbances', Current Paediatrics. 2003 13: pp. 520-528.

[3] Goldberg, A. Hammerman, H. Petcherski, S. Nassar, M. Zdorovyak, A. Yalonetsky, S. et al. (2006) 'Hyponatremia and long-term mortality in survivors of acute ST-elevation myocardial infarction', Arch Intern Med. 166: pp. 781-786.

[4] Sharkawy, A. M. Sahota, O. Maughan, R. J. and Lobo, D. N. (2014) 'The pathophysiology of fluid and electrolyte balance in the older adult surgical patient', Clinical Nutrition. 33: pp. $6-13$.
[5] Zaman, M. M. Choudhury, S. R. Ahmed, J. Numan, S. M. Islam, S. M. and Yoshiike, N. (2004) 'Non biochemical risk factors for cardiovascular disease in general clinic-based rural population of Bangladesh', Journal of Epidemiology. 14: pp. 63-68.

[6] Ahmed, S (2014) 'Electrolyte Imbalance in Admitted Diabetic Patients: Patterns and Factors Responsible- A Cross Sectional Study', KYAMC Journal, 5 (1), pp. 458-466.

[7] Haque, H. et al. (2012) 'Pattern of Electrolyte Imbalance in Hospitalized Diabetic Patients: Experience in a Tertiary Care Hospital', BIRDEM Medical Journal, 2 (1), pp. 14-18.

[8] Siragy, H. M. (2006) 'Hyponatremia, fluid-electrolyte disorders, and the syndrome of inappropriate antidiuretic hormone secretion: diagnosis and treatment options', Endocrine practice, 12 (4), pp. 446-57.

[9] Clayton, J. A., Le Jeune, I. R. and Hall, I. P. (2006) 'Severe hyponatraemia in medical in-patients: Aetiology, assessment and outcome', Qjm, 99 (8), pp. 505-51.

[10] Boysen, S. R. (2008) 'Fluid and Electrolyte Therapy in Endocrine Disorders: Diabetes Mellitus and Hypoadrenocorticism', Veterinary Clinics of North America Small Animal Practice, 38 (3), pp. 699-717.

[11] Vryonidou, A. et al. (2018) 'Hypokalemia: a clinical update', Endocrine Connections, 7 (4), pp. 135-146.

[12] Fraser, R. (1984) 'Disorders of the adrenal cortex: their effects on electrolyte metabolism', Clinics in Endocrinology and Metabolism, 13 (2), pp. 413-430.

[13] Kumar, V. (2018) 'Case Report A Case of Diabetic Ketoacidosis Presenting with Hypernatremia, Hyperosmolarity, and Altered Sensorium', Case Reports in Endocrinology, 2018, pp. 1-4.

[14] Das gupta, A., Saikia, U. and Sarma, D. (2012) 'Hypomagnesemia in type 2 diabetes mellitus', Indian Journal of Endocrinology and Metabolism, 16 (6), p. 1000.

[15] Sales, C. H. and Pedrosa, L. de F. C. (2006) 'Magnesium and diabetes mellitus: Their relation', Clinical Nutrition, 25 (4), pp. 554-562.

[16] Qureshi, S. et al. (2015) 'A severe case of ectopic ACTH presenting with Cushing's syndrome with hypokalaemia, hypomagnesemia, hypophosphatemia, hyponatraemia and hypocalcaemia', Endocrine Abstracts. BioScientifica. 